predictors of outcome of SE. (Neligan A, Shorvon SD. Prognostic factors, morbidity and mortality in tonic-clonic status epilepticus: a review. Epilepsy Res 2011 Jan;93(1):1-10). In otherwise normal children with focal epilepsy, SE has no significant effect on longterm intellectual and seizure outcome. (Camfield P, Camfield C. Pediatrics 2012 Sep;130(3):e501-6).

\title{
CLINICAL, BIOCHEMICAL, AND MOLECULAR STUDIES AND TREATMENT OF PYRIDOXINE-DEPENDENT EPILEPSY
}

Researchers at Autonomous University of Madrid, and other centers in Spain studied the clinical, biochemical, and genetic spectrum of pyridoxine-dependent epilepsy (PDE) in 12 patients with the clinically proven diagnosis. Onset of seizures varied from neonatal to first months of life. Seizures were focal or multifocal, clonic or myoclonic, and generalized tonic; $50 \%$ had status epilepticus. Seizures were controlled transiently with conventional AEDs for 15 or more days in 8 of 12 patients, leading to a delay in diagnosis. The effective dose of pyridoxine to suppress seizures ranged from 10 to 30 $\mathrm{mg} / \mathrm{kg} /$ day. Neurologic symptoms in addition to seizures included hypotonia, irritability, and psychomotor retardation. EEG abnormalities were variable and included focal or multifocal discharges, burst-suppression pattern, and generalized slowing. A normal EEG in one patient does not rule out the diagnosis. All EEGs became normal after pyridoxine therapy. MRI abnormalities, mainly posterior fossa, included mega cisterna magna, Dandy Walker syndrome, ventriculomegaly, and corpus callosum dysgenesis. Six patients followed for more than 5 years show cognitive dysfunction and borderline or mildly retarded IQs. Delay in treatment and dysgenesis of the corpus callosum are risk factors for neurodevelopmental delay. Treatment with pyridoxine does not normalize the IQ. Urine levels of a-aminoadipic semialdehyde (a-AASA) and plasma/CSF levels of pipecolic acid (PA) are diagnostic biomarkers. Genetic analysis of these Spanish patients showed 12 mutations, 7 novel, and different from other populations. (Perez B, GutierrezSolana LG, Verdu A, et al. Clinical, Biochemical, and molecular studies in pyridoxinedependent epilepsy: Antisense therapy as possible new therapeutic option. Epilepsia 2013 Feb;54(2):239-48). (Response: Dr Belen Perez. E-mail: bperez@cbm.uam.es).

COMMENT. PDE should be considered in any infant with intractable seizures, including patients with MRI abnormalities such as corpus callosum dysgenesis or Dandy Walker syndrome. The long-term outcome of PDE was poor in a Dutch PDE cohort of 14 patients. (Bok LA, Halbertsma FJ, Houterman S, et al. Dev Med Child Neurol 2012 Sep;54(9):849-54). EEG background and epileptiform activity were not correlated with outcome. Delayed initiation of pyridoxine and the association of corpus callosum abnormalities were significantly associated with unfavorable neurodevelopmental outcome, findings similar to the Spanish experience.

\section{PDE WITH MOLYBDENUM COFACTOR DEFICIENCY}

PDE with molybdenum cofactor deficiency is reported in 2 siblings. Molecular investigations revealed a homozygous mutation in the MOCS2 gene. Pyridoxine supplementation is recommended in patients diagnosed with molybdenum cofactor or 
sulfite oxidase deficiencies. (Struys EA, Nota B, Bakkali A, et al. Pediatrics 2012 Dec;130(6):e1716-9).

\section{ATTENTION DEFICIT AND AUTISTIC DISORDERS}

\section{FUNCTIONAL MAGNETIC RESONANCE IMAGING STUDIES OF INHIBITION AND ATTENTION IN ADHD}

Researchers at King's College, London; Kyushu University, Japan; and Barcelona, Spain conducted a meta-analysis of functional magnetic resonance imaging (fMRI) in ADHD during inhibition and attention tasks, with reference to age and effects of long-term use of stimulant medication. Twenty-one data sets were included in the inhibition meta-analysis ( 7 adult and 14 pediatric samples), and 13 data sets in the attention meta-analysis ( 2 adult and 11 pediatric samples). Combined, the inhibition studies included 287 patients with ADHD and 320 healthy controls. Compared to controls, patients with ADHD showed reduced activation for inhibition in the right inferior frontal cortex, supplementary motor area, anterior cingulate cortex, and striatothalamic areas, and reduced activation for attention in the right dorsolateral prefrontal cortex, posterior basal ganglia, thalamic and parietal regions. Long-term stimulant medication use was associated with more normal right caudate activation during the attention domain. For the inhibition meta-analysis only, the supplementary motor area and basal ganglia were under-activated in children with ADHD, while the inferior frontal cortex and thalamus were under-activated solely in adults with ADHD relative to controls. (Hart H, Radua J, Nakao T, Matai-Cols D, Rubia K. Meta-analysis of functional magnetic resonance imaging studies of inhibition and attention in attentiondeficit/hyperactivity disorder. JAMA Psychiatry 2013 Feb;70(2):185-198). (Response: Dr Katya Rubia. E-mail: katya.rubia@kcl.ac.uk).

COMMENT. In conclusion, patients with ADHD have cognitive dysfunctions in right fronto-basal ganglia-thalamic networks, and long-term stimulant medication is associated with normalization of right caudate deficits during attention. In addition to inhibition and attention studies, future meta-analyses should include other compromised functions such as timing and motivation. In the present study, for the inhibition domain, go/no-go, stop tasks and Stroop tasks were used, and for the attention domain, tasks that measured visuospatial selective attention, sustained attention, and flexible attention.

\section{SELECTIVE DOPAMINE REUPTAKE INHIBITOR AND ADHD}

ADHD treatments act as dual norepinephrine (NE) and dopamine (DA) reuptake inhibitors (psychostimulants) or selective NE reuptake inhibitors (SNRIs). Benztropine analogs (AHN2-005) act as highly selective DA reuptake inhibitors while lacking the abuse potential of psychostimulants. A cognition-enhancing dose of AHN 2-005 increased levels of DA and NE in the prefrontal cortex (PFC) and may be effective in the treatment of ADHD associated with PFC dysfunction. (Schmeichel BE et al. Neuropharmacology 2013 Jan;64:321-8). Neuropharmacology and fMRI studies should expand the development of new, more effective agents in the treatment of ADHD. 